\title{
Practical implementation analysis of the fractal dimension calculating algorithm for the medical images by the example of neutrophil nuclei
}

\author{
Semen A. Naydenov ${ }^{1 *}$, Pavel A. Naydenov ${ }^{2}$, and Elena V. Shevchenko ${ }^{1}$ \\ ${ }^{1}$ Irkutsk State Medical University, 1 Krasnogo Vosstaniya st., Irkutsk 664003, Russia \\ ${ }^{2}$ Irkutsk State University, 1 Karl Marx st., Irkutsk 664003, Russia \\ *e-mail: semen.naid@yandex.ru
}

\begin{abstract}
This paper presents the results of estimating the fractal dimension of neutrophil nuclei to determine their functional state. Calculations were carried out by a specially developed software-implemented algorithm. The study has shown that the fractal theory can be applied to automate medical image processing. (C) 2020 Journal of Biomedical Photonics \& Engineering.
\end{abstract}

Keywords: fractals; image processing; neutrophils; medical images.

Paper \#3346 received 15 Jan 2020; revised manuscript received 15 Mar 2020; accepted for publication 17 Mar 2020; published online 24 Mar 2020. doi: 10.18287/JBPE20.06.010304.

\section{Introduction}

Nowadays, investigations into medical images have become well established in clinical diagnostics of various pathologies. Thus, the use of X-ray radiation, acoustic waves, laser radiation, microscopic and computer-assisted studies for obtaining images, has considerably expanded the possibilities of contemporary healthcare [1, 2]. Development and implementation of a detailed mathematical image processing methodology, be it microphotography or an X-ray picture, by utilizing electronic computer systems, will considerably increase processing efficiency [3]. One of the numerical methods to process nonlinear images, including medical images, is estimating their fractal dimension $[4,5]$. This method is actively used both in Russia and internationally [6-10]. However, to our knowledge, there has not been any research on utilizing fractal analysis for neutrophil images, which can testify the relevance of this study.

Current clinical practice makes wide use of White Blood Cell Differential Count - percentage ratio of various leukocyte types, especially different neutrophil forms, when they are counted in a stained blood smear under a microscope.

Neutrophil granulocytes (neutrophil leukocytes, neutrophils) is the most numerous group of leukocytes. The reference values of their blood level are within 2.0 to $5.5 \times 10^{9}$ per $1 \mathrm{~L}$ (which makes up from 48 to $78 \%$ of the total leukocyte number). The nucleus of a mature segmented neutrophil contains 3-5 segments connected by thin filaments. In blood, there are neutrophils of three types of maturity: metamyelocytes (juvenile neutrophils), band neutrophils and segmented neutrophils. Metamyelocytes and band neutrophils qualify as young cells. Under normal conditions, the metamyelocyte level does not exceed $0.5 \%$ and is characterized with a kidney bean shaped nucleus. Band neutrophils under normal conditions make up from 1 to $6 \%$ of the total number of leukocytes, have an unsegmented nucleus often of a horseshoe shape or $S$-shape. Increased percentage of metamyelocytes and band neutrophils may indicate the presence of an acute pyoinflammatory process or acute vast blood loss. This is caused by accelerated hemopoiesis process and by replacement of the lost cells with younger forms [11].

Hence, the neutrophil classification and percentage calculation for each type present an important clinical test. Moreover, the cell type and age are directly connected with the nucleus structure, particularly with its segmentation, which in today's clinical practice is determined by physicians visually and subjectively. In addition, microscopic analysis of blood smears in conditions of an ordinary hospital is now quite laborious due to a large number of patients.

Therefore, the aim of this study is to develop an automated method for medical image processing based on fractal analysis, which could cover the microscopic analysis of blood smears as well.

\section{Materials and Methods}

The materials for this study were fixed and routine stained blood smears in patients of the Surgery Department of Irkutsk Research Center of Surgery and Traumatology (Russia). Blood was collected before and after surgery. The blood smears were stained by 



Fig. 1 Neutrophils in the blood smear: a) Metamyelocyte, b) Band neutrophil, c) Segmented neutrophil.

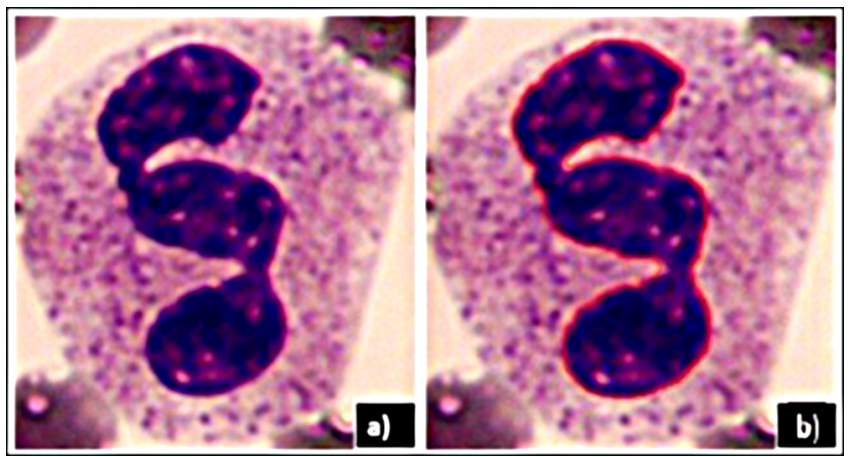

Fig. 2Iimage pre-processing: a) before processing, b) after processing.

Romanowsky-Giemsa solution for 20 minutes. The protocols of the investigation were approved by the medicine ethical committee of Irkutsk State Medical University.

The prepared smears were viewed and microphotographed by the Altami-163t microscope equipped with the Levenhuk C-Series digital camera with the resolution of $2048 \times 1546 \mathrm{px}$. The cells were viewed at $2000 \times$ magnification.

The fractal dimension was calculated by a specially developed programmable algorithm.

For the purpose of sampling, the cells were classified into metamyelocytes, band granulocytes and segmented granulocytes. The neutrophils were classified according to the morphological characteristics of the granulocytic lineage cells presented below [12].

Metamyelocytes (Fig. 1a) has the cell size of 10 to $16 \mu \mathrm{m}$. The nucleus is of kidney-bean shape and lilac color. The cytoplasm is pale pink, smoke-grey or light blue. Inside, the cell has neutrophil granularity evenly spread over the cytoplasm.

Band neutrophils (Fig. 1b) has the cell size is 9 to $12 \mu \mathrm{m}$. The nucleus is of curved band shape and of purple color. The chromatin has a crude structure. The cytoplasm is pink with a purple shade. Inside, it is characterized by neutrophil granularity, often unevenly spread over the cytoplasm.

Segmented neutrophils (Fig. 1c) has the cell size is 9 to $12 \mu \mathrm{m}$. The nucleus is of irregular shape, polymorphic, segmented (with 2 to 5 lobes) and bright purple. Between the lobes there are thin connecting filaments. The cell cytoplasm is pink or pink purple with neutrophil granularity.

The fractal dimension calculation based on the covering method [4,5] was performed by specially developed algorithm that is described, step-by-step, below.

1. The image is pre-processed by means of a graphic editor as shown in Fig. 2.

2. The fractal dimension is calculated by specially developed software (Fig. 3) written and implemented using Borland Delphi 7 platform.

Below is the general equation for calculating fractal dimension [4]:

$$
D=-\lim _{\varepsilon \rightarrow 0} \frac{\log (N(\varepsilon))}{\log \left(\frac{1}{\varepsilon}\right)}
$$

where $D$ is fractal dimension, $N$ is the number of boxes $\mathcal{E}, \mathcal{E}$ is the box size.

Image pre-processing was performed by the means of Adobe Photoshop CS 6, Stroke tool, 2 px stroke width, red color (R-255, G-0, B-0; \#ff0000). The $\varepsilon$ box size was set in px (5 px and more).

The program includes a set of commands that perform the following tasks:

1. Uploading the image to be processed from a prepared file. 


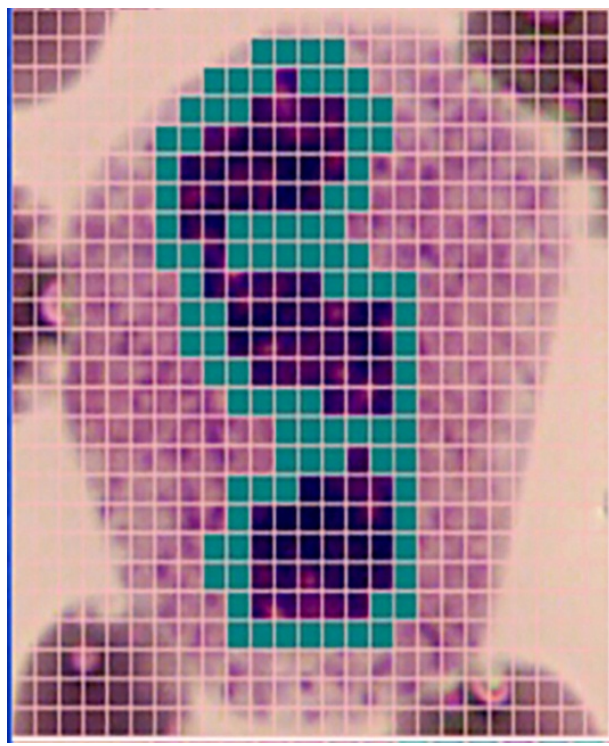

\begin{tabular}{|l|l|}
\hline $\begin{array}{c}\text { Box Size } \\
\text { in pix } \\
\text { (a) }\end{array}$ & $\begin{array}{c}\text { Cell } \\
\text { number } \\
\text { (N) }\end{array}$ \\
\hline 5 & 402 \\
\hline 10 & 132 \\
\hline 15 & 120 \\
\hline 20 & 90 \\
\hline 25 & 70 \\
\hline 30 & 58 \\
\hline 35 & 43 \\
\hline 40 & 41 \\
\hline 45 & 35 \\
\hline 50 & 32 \\
\hline
\end{tabular}

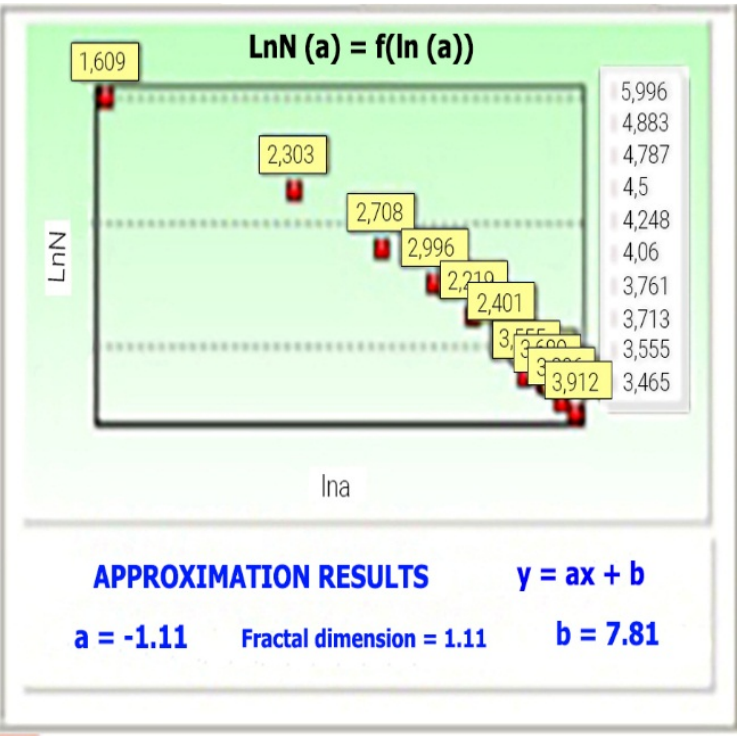

Fig. 3 Calculation of fractal dimension by means of specially developed software.

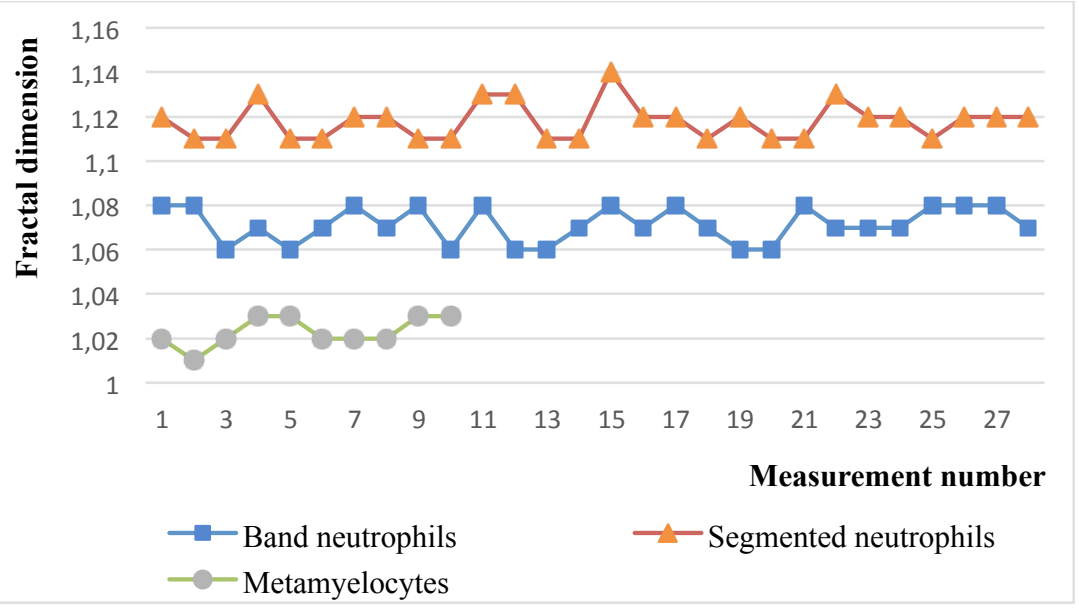

Fig. 4 Comparison of the values of fractal dimension for neutrophil nuclei.

2. Building matrices above the uploaded image. The matrices consist of square boxes of a certain size measured in pixels.

3. Along with building the matrices, the program is counting the boxes whose area contains at least one pixel of the outline under examination.

4. Building a graph based on the calculated data with simultaneous calculating the fractal dimension.

\section{Results}

The results of calculating the neutrophil fractal dimension are presented in Fig. 4. The $\mathrm{x}$-axis represents the measurement number where each measurement corresponds to a new sample. Consequently, the following types of neutrophils were examined: 28 band neutrophils, 28 segmented neutrophils and 10 metamyelocytes.

The graph shows no crossing of fractal dimension values for neutrophils belonging to three various groups according to their morphological characteristics: for band neutrophils, the nucleus fractal dimension is ranging between 1.06 and 1.08. For segmented neutrophils, the nucleus fractal dimension is ranging between 1.11 and 1.14. For metamyelocytes, the nucleus fractal dimension is ranging between 1.01 and 1.03. The confidence interval was estimated using a standard method for small samples.

The obtained data were processed through estimating Student's coefficient for independent variables. Comparison of the critical and empirical t-tests has demonstrated statistical significance of differences between the nucleus fractal dimension of metamyelocytes, segmented neutrophils and band neutrophils.

This result reflects the fact that fractal dimension is not dependent on the nucleus size of the samples but on their shape. The fractal method is able to estimate the extent of the object 'unevenness, i.e., in fact, to numerically determine and distinguish what a physician determines intuitively examining the sample with a microscope. The suggested algorithm for estimating the nuclear fractal dimension is able to automatically determine the neutrophil types and, consequently, to 
divide the neutrophils into band neutrophils, segmented neutrophils and metamyelocytes.

\section{Conclusion}

The study has shown the possibility to apply the fractal theory for automating medical image processing. A new algorithm has been developed for computer-assisted calculation of the fractal dimension for medical images. The fractal dimension of the neutrophil nuclei is dependent on their age:

- for metamyelocytes, the values of the fractal dimension are ranging between 1.01 and 1.03;
- for band neutrophils, they are ranging between 1.06 and 1.09 ;

- for segmented neutrophils, they are ranging between 1.11 and 1.14.

Fractal dimension has proved to be a sufficient parameter for determining the neutrophil type and is independent of the patient's individual characteristics.

\section{Disclosures}

All authors declare that there is no conflict of interests in this paper.

\section{References}

1. V. V. Goryunova. T. I. Goryunova. I. I. Kukhtevich, and T. T. Polyakova, "Processing medical images in graph databases and patient identification," Mezhdunarodnyy studencheskiy nauchnyy vestnik 4(9), 1390-1393 (2017) [in Russian].

2. S. V. Ablameyko, A. M. Nedzved, Processing optical images of cell structures in medicine, OIPI NAN Belarusi, Minsk (2005) [in Russian].

3. N. N. Chernov, A. P. Samoylenko, and A. V. Pribylskiy, "A technique to increase the informative value of X-ray image pre-processing," Modelirovaniye, optimizatsiya i informatsionnyye tekhnologii 6(1), 6-20 (2018) [in Russian]..

4. A. K. Ostapchuk, V. E. Ovsyannikov, Application of fractal theory in mathematical modelling and equipment, Izdetelstvo Kurganskogo gosudarstvennogo universiteta, Kurgan (2009) [in Russian].

5. P. Nesterov, "Calculating Minkowskian fractal dimension for 2D-imaging," Habr (accessed 15.12.2018) [in Russian].

6. A. I. Povoroznyuk, A. V. Emelianova, “A system of medical image processing in radiology by means of fractal analysis," Vestnik NTU "KhPI" 35, 147-151 (2014) [in Russian].

7. G. S. Simonyan, A. G. Simonyan, "Fractality of biological systems. II Fractality of cells and cell ensembles," Mezhdunarodnyy zhurnal prikladnykh i fundamentalnykh issledovaniy 3(2), 268-271 (2016) [in Russian].

8. P. Bose, N. T. Brockton, K. Guggisberg, S. C. Nakoneshny, E. Kornaga, A. C. Klimowicz, M. Tambasco, and J. C. Dort, "Fractal analysis of nuclear histology integrates tumor and stromal features into a single prognostic factor of the oral cancer microenviroment," BMC Cancer 15(1), 409-418 (2015).

9. S. Bhandari, S. Choudannavar, E. R. Avery, P. Sahay, and P. Pradhan "Detection of colon cancer stages via fractal dimension analysis of optical transmission imaging of tissue microarrays (TMA)," Biomedical Physics \& Engineering Express 4(6), 65-75 (2018).

10. K. Metze, R. Adam, J. B. Florindo, "The fractal dimension of chromatin - a potential molecular marker for carcinogenesis, tumor progression and prognosis," Expert Review of Molecular Diagnostics 19(4), 299-312 (2019).

11. Yu. I. Afanasyev, N. A. Yurina, and E. F. Kotovskiy, Histology, cytology and embryology, Meditsina, Moscow (2002) [in Russian].

12. Laboratory diagnostics (accessed 29.11.2018).

13. P. S. Aleksandrov, P. S. Uryson, Memoir on compact topological spaces, Fizmatlit, Moscow (2009) [in Russian].

14. B. Mandelbrot, Fractal geometry of nature, Institut Kompyuternykh Issledovaniy, Moscow (2002) [in Russian]. 